\title{
RIQUEZA DE AVES DA FAZENDA SANTA RITA, REGIÃO DOS CAMPOS GERAIS, PALMEIRA, PARANA, BRASIL
}

\author{
Luiz dos Anjos 1 \\ Vinalto Graf ${ }^{2}$
}

\begin{abstract}
RICHNESS OF BIRDS OF SANTA RITA FARM, CAMPOS GERAIS REGION, PALMEIRA COUNTY, PARANÁ, BRAZIL. Two hundred and eighty eight bird species were registered in Santa Rita Farm, east of Paraná State in Brazil (28 $\left.18^{\circ} \mathrm{S}, 49^{\circ} 48^{\prime} \mathrm{W}\right)$, from February 1990 to January 1991. Eighty four species were registered in all 12 months and eighty three were registered once during this period. October was the richest in number of species (220) and July was the poorest (137). The natural landscape of the Santa Rita farm is composed of open field with several patches of araucaria forest (from 10 to 40 ha) and some small areas of marshland. Two hundred and eight species occurred only in one of these habitats: 163 in the forest, 35 in the open field and 10 in the marshland. The others often occurred in both forest and open field; there is a gradient of occurrence between them. Columba speciosa Gmelin, 1789; C. maculosa Temminck, 1813; Dolichonyx oryzivorus (Linnaeus, 1758) and Sporophila melanogaster (Pelzeln, 1870) were new field registers in Parana State. Because of its great number of bird species (some endangered species) and its landscape, composed of well preserved habitats, Santa Rita farm is shown as a strategic area for conservation.
\end{abstract}

KEY WORDS. Birds, richness, distribution, Paraná, Brazil

A região dos Campos Gerais está localizada no centro leste do Estado do Paraná ocupando principalmente os municípios de Ponta Grossa, Palmeira, Campo Largo, São João do Triunfo, Telêmaco Borba, Castro e Tibagi (aproximadamente entre $24^{\circ}$ e $26^{\circ} \mathrm{S}$ e $49^{\circ} 30^{\prime}$ e $50^{\circ} 30^{\prime} \mathrm{W}$ ). Embora a maior parte da fitofisionomia natural desta região seja composta por campo, existem manchas naturais de floresta de araucária dispersas e isoladas entre si denominadas capões. Tanto na porção oriental como na ocidental dos Campos Gerais, os capões tendem a ser maiores e ligados até formarem uma floresta contínua.

Os Campos Gerais vem sofrendo intensa atividade antrópica, já salientada na década de sessenta por KLEIN (1960). Neste processo o ambiente natural foi se restringindo, só podendo ser atualmente visualizado em determinados locais como a Fazenda Santa Rita.

1) Departamento de Biologia Animal e Vegetal, Universidade Estadual de Londrina, Caixa Postal 6001, 86051-970 Londrina, Paraná, Brasil. Bolsista do CNPq.

2) Departamento de Zoologia, Universidade Federal do Paraná, Caixa Postal 19020, 81531-970 Curitiba, Paraná, Brasil. 
O objetivo do presente estudo é analisar a avifauna que ocorre na Fazenda Santa Rita localizada na borda oriental da região dos Campos Gerais. A análise é realizada em relação à distribuição sazonal, à distribuição nos habitats, à riqueza e à composição específicas.

Em decorrência de observações esporádicas, existem muitos registros de espécies de aves que ocorrem na região dos Campos Gerais, inclusive na própria Fazenda Santa Rita levantados por LOPES \& SCHERER-NETO. Entretanto, nenhum levantamento contínuo foi realizado. A exceção é o levantamento de aves do Parque Estadual de Vila Velha feito por SCHERER-NETO; ANJOS \& STRAUBE, realizado ao longo de um ano quando foram registradas 162 espécies.

\section{MATERIAL E MÉTODOS}

\section{ÁREA DE ESTUDO}

O trabalho de campo foi desenvolvido em 2000 ha da Fazenda Santa Rita, Palmeira, Paraná $\left(28^{\circ} 18^{\prime} \mathrm{S}, 49^{\circ} 48^{\prime} \mathrm{W}\right)$. Nesta fazenda, que está localizada em um planalto a aproximadamente 1000 metros do nível do mar, ocorrem vários capões com 10 a 40 ha dispersos em campo e uma floresta contínua de aproximadamente 1000 ha constituídos por floresta de araucária. Além do campo e da floresta existem pequenas áreas de brejo.

Nas áreas com floresta a Araucaria angustifolia Bert. O. Kuntze é a espécie arbórea dominante sendo também muito comuns Campomanesia xantocarpha Berg, Eugenia uniflora Linnaeus, Schinus terebenthifolius Raddi, Jacaranda puberola Chamissou, Piptocarpha angustifolia Dusen, Nectandra lanceolata (Nees \& Martius) e Cedrella fissilis Velloso. No campo predominam as gramíneas como Andropogon selloanus (Hack), Axonopus brasiliensis (Sprengel) e Paspalum notatum Flügge. Nas pequenas áreas de brejo predominam ciperáceas e juncáceas.

\section{MÉTODOS}

Foram realizadas amostragens mensais de quatro dias de permanência em campo de fevereiro de 1990 a janeiro de 1991. O tempo de observação em campo em cada uma das doze amostragens foi de aproximadamente 40 horas totalizando 470 horas para todo o estudo.

A identificação das espécies de aves em campo baseou-se no contato visual e/ou auditivo. Além do registro anotou-se para cada espécie em cada amostragem o (s) habitat (s) em que foi observada. Foram reconhecidos cinco habitats: floresta, campo, brejo, plantação e jardim. Jardim corresponde às áreas próximas às casas dos moradores da região. Durante a amostragem não se seguia uma trilha padrão mas procurava-se percorrer todos os habitats (VIELLIARD \& SILVA, 1990). 


\section{RESULTADOS E DISCUSSÃO}

\section{DISTRIBUIÇÃO SAZONAL}

A flutuação da riqueza específica ao longo do ano mostra que o número de espécies começa a aumentar a partir do final do inverno (setembro) diminuindo no final da primavera (dezembro) e mais ainda no outono (maio) (Fig. 1). O mês de julho foi o mais pobre em número de espécies registradas (137) e o mês de outubro o mais rico (220).

\section{NÚMERO DE ESPÉCIES AO LONGO.DO ANO}

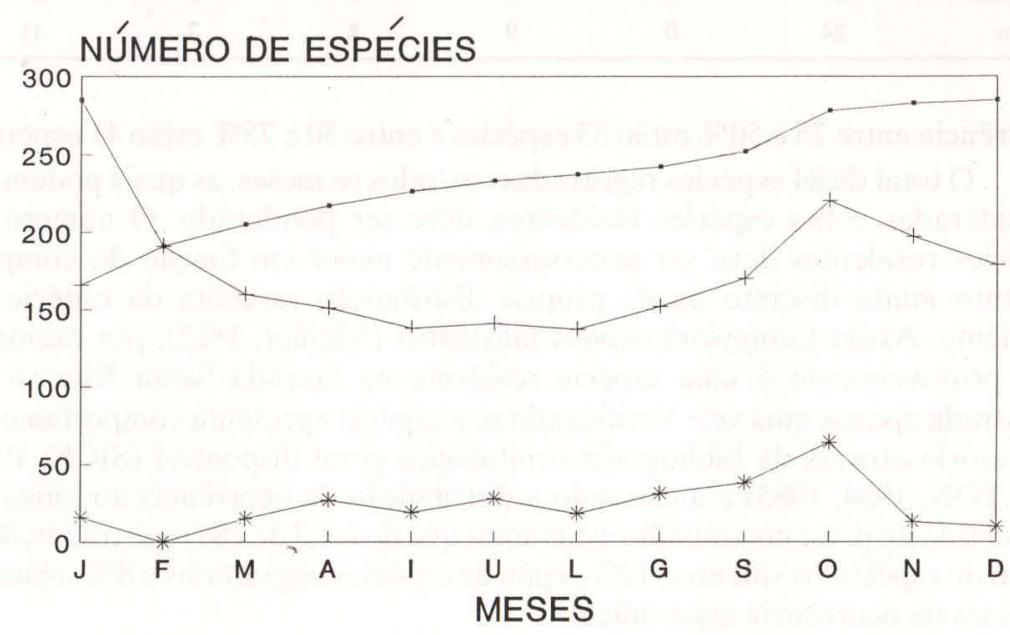

- número acumulado + esp. encontradas * esp. não encontradas

Fig. 1. Número de espécies encontradas em cada mês de amostragem, de não encontradas no mês anterior e acumuladas al longo do ano.

A riqueza específica foi mais alta de setembro a fevereiro em função da presença de espécies migratórias e do período de atividade reprodutiva (que torna as aves mais evidentes no ambiente). Foi justamente mais baixa de março a agosto na ausência das espécies migratórias e com o comportamento mais discreto das aves fora do período reprodutivo. Não considerando estas duas variáveis relacionadas com o ciclo biológico o esforço de trabalho em campo foi regular pois o número de espécies não registradas na visita anterior se manteve baixo (Fig. 1).

Podemos determinar a freqüência de ocorrência ao longo do ano que é o número de meses em que a espécie foi registrada (expresso em porcentagem) (Tab. I). Um total de 111 espécies ( $39 \%$ da avifauna) apresentaram freqüência de ocorrência superior a $75 \%$ e, dentre estas, 84 (29\% da avifauna) ocorreram nos doze meses com freqüência de ocorrência igual a $100 \%$. Por outro lado 83 espécies (29\% da avifauna) só foram registradas em três meses ou menos e destas 39 (14\% da avifauna) tiveram apenas um registro. Com freqüência de 
Tabela I. Número de espécies por classes de freqüência de ocorrência geral e por habitat.

\begin{tabular}{lcccccc}
\hline & \multicolumn{6}{c}{ Freqüência de ocorrência até: } \\
\cline { 2 - 7 } & $25 \%$ & $25-50 \%$ & $50-75 \%$ & $75-100 \%$ & $100 \%$ & Total \\
\hline Geral & 83 & 53 & 41 & 111 & 84 & 288 \\
Floresta & 8 & 14 & 11 & 186 & 181 & 219 \\
Campo & 14 & 13 & 10 & 57 & 48 & 94 \\
Brejo & 9 & 5 & 2 & 11 & 10 & 27 \\
Plantação & 17 & 6 & 0 & 1 & 1 & 24 \\
Jardim & 24 & 0 & 9 & 8 & 7 & 41 \\
\hline
\end{tabular}

ocorrência entre 25 e $50 \%$ estão 53 espécies e entre 50 e $75 \%$ estão 41 espécies.

O total de 84 espécies registradas em todos os meses, as quais podem ser consideradas como espécies residentes, deve ser ponderado. O número de espécies residentes deve ser necessariamente maior em função do comportamento muito discreto ou da própria distribuição rarefeita da espécie no ambiente. Assim Campylorhamphus falcularius (Vieillot, 1822), por exemplo, que provavelmente é uma espécie residente na fazenda Santa Rita só foi registrada apenas uma vez. Verificando se a espécie apresenta comportamento migratório através da bibliografia ornitológica geral disponível (SICK, 1985; BELTON, 1984, 1985) e analisando a distribuição da ocorrência ao longo do ano obtida no presente trabalho estimamos que do total de 288 registradas, $80 \%$ sejam de espécies residentes, $12 \%$ sejam de espécies migratórias e $8 \%$ sejam de espécies de ocorrência esporádica.

\section{DISTRIBUIÇÃO NOS HABITATS}

Podemos determinar a freqüência de ocorrência por habitat que é o número de meses em que a espécie foi registrada em cada habitat identificado neste trabalho (expressa em porcentagem) (Tab. I).

A floresta foi o habitat com o maior número de espécies registradas (219 ou $76 \%$ da avifauna). Além disto a maioria das espécies apresentou uma alta relação com este habitat pois $181(83 \%)$ apresentaram $100 \%$ de ocorrência e $161(74 \%)$ ocorreram somente neste habitat, podendo ser consideradas como exclusivas. Apenas 14 espécies apresentaram freqüência de ocorrência inferior a $50 \%$ na floresta (Tab. I).

O campo foi o segundo habitat mais rico com 94 espécies registradas (33\% da avifauna). Mas apenas $48(52 \%)$ apresentaram 100\% de ocorrência e $34(37 \%)$ ocorreram somente neste habitat.

Entretanto muitas espécies apresentaram registros na floresta e no campo. Dentre estas que ocorrem nestes dois habitats geralmente as não Passeriformes são espécies campestres que ocupam a floresta como local de pouso ou de nidificação e as Passeriformes são espécies florestais que ocupam eventualmente o campo como local de alimentação. A exceção é a família 
Fringillidae cuja maioria dos representantes vivem no campo.

Theristicus caudatus (Boddaert, 1783) passa o dia alimentando-se no campo, mas procura as araucárias dos capões para o pouso noturno e para a nidificação (SCHERER-NETO, 1982; LOPES, 1982). Na área de estudo esta espécie, normalmente encontrada durante o dia em pequenos grupos, se reúne no final da tarde em bandos de aproximadamente 200 indivíduos em três ou quatro araucárias (Lopes, com. pes.). Polyborus plancus (Miller, 1777) e Colaptes campestris (Vieillot, 1818) passam a maior parte do tempo no campo mas geralmente dormem e nidificam na floresta.

Pitangus sulphuratus (Linnaeus, 1766), Myiophobus fasciatus (Temminck, 1822) e Thraupis sayaca Linnaeus 1766 são exemplos de Passeriformes que ocupam normalmente a borda da floresta mas que visitam com maior ou menor freqüência o campo durante o forrageamento (ANJOS, 1984; 1990). Existem ainda espécies como Elaenia obscura Zimmer, 1941 que, na área de estudo, não foi observada nem no interior da floresta nem no campo adjacente permanecendo na borda.

Assim há um gradiente de ocupação da avifauna em relação à floresta $\mathrm{e}$ ao campo. Este gradiente de ocupação varia conforme os estágios de sucessão vegetal da borda da floresta. Como já analisado em um capão de floresta de araucária (ANJOS, 1990), se existem muitos estágios na borda, com a altura da vegetação diminuindo gradativamente, então a avifauna é rica em número de espécies; se existem poucos estágios, com a altura da vegetação diminuindo abruptamente, então a avifauna é mais pobre em número de espécies.

O brejo foi o ambiente natural com menor riqueza específica: 27 espécies ou $10 \%$ da avifauna. Um total de $10(37 \%)$ destas espécies ocorreram somente neste habitat. As outras, que apresentaram freqüência de ocorrência geralmente inferior a 50\%, constituem-se principalmente de Passeriformes campestres que visitam irregularmente o brejo. Isto demonstra que a avifauna que ocupa o brejo é restrita e particular deste habitat como Casmerodius albus (Gmelin, 1789), Amazonetta brasiliensis (Gmelin, 1789) e Gallinago undulata (Boddaert, 1783).

Plantação e jardim são habitats criados em função da atividade antrópica. A maioria das aves estão na verdade de passagem por estes habitats aproveitando algum recurso alimentar disponível o que parece estar demonstrando o elevado número de espécies com baixa freqüência de ocorrência nestes habitats. Crotophaga ani Linnaeus, 1758 e Satrapa icterophrys (Vieillot, 1818) casualmente só foram registradas nestes habitats embora sejam espécies de campo ou de borda de floresta. Passer domesticus (Linnaeus, 1758) e Tyto alba (Gray, 1769), por outro lado, apresentam muita relação com o jardim.

\section{RIQUEZA E COMPOSIÇÃO ESPECÍFICAS}

Um total de 288 espécies de aves foram registradas na fazenda Santa Rita sendo apresentadas na tabela II seguindo-se a taxonomia de MEYER DE SCHAUENSEE (1970). Três espécies não tiveram segura identificação embora tenham sido consideradas na lista geral:Phaetornis squalidus (Temminck, 1822), Philydor atricapillus (Wied, 1821) e Elaenia flavogaster (Thumberg, 1822). Por 
outro lado quatro foram pela primeira vez registradas no Estado do Paraná: Columba speciosa Gmelin, 1789, Columba maculosa Temminck, 1813, Dolichonyx oryzivorus (Linnaeus, 1758) e Sporophila melanogaster (Pelzeln, 1870). Embora a distribuição geográfica destas espécies incluísse o Estado do Paraná (MEYER DE SCHAUENSEE, 1970) não haviam ainda registros de campo.

Dezesseis espécies foram registradas através de observações esporádicas ao longo de alguns anos antes da realização do presente trabalho (LOPES \& SCHERER-NETO, com. pes.). Algumas destas espécies não foram por nós registradas por serem possivelmente muito raras na região: Elanoides forficatus (Linnaeus, 1758), Anthracothorax nigricollis (Vieillot, 1817), Suiriri suiriri (Vieillot, 1818), Anthus correndera (Vieillot, 1818), Molothrus badius (Vieillot, 1819), Schistochlamys ruficapillus (Vieillot, 1817) e Paroaria coronata (Miller, 1776).

As outras espécies registradas pelos autores acima mencionados são raras em virtude de só aparecerem em anos atípicos com alta pluviosidade e formação de lagoas temporárias: Mycteria americana (Linnaeus, 1758), Plegadis chihi (Vieillot, 1817), Sarkidiornis melanotos (Ihering \& Ihering, 1907), Cairina moschata (Linnaeus, 1758), Jacana jacana (Linnaeus, 1758), Calidris fuscicollis (Vieillot, 1819), Ceryle torquata (Linnaeus, 1766) e Culicivora caudacuta (Vieillot, 1818). A formação do platô assintótico, obtido através do número acumulado de espécies, corrobora que as acima citadas podem ser consideradas como de ocorrência esporádica (Fig. 1).

Considerando-se a área estudada a riqueza específica de 303 espécies de aves da fazenda Santa Rita se mostra alta. Aproximadamente na mesma latitude LANGE \& STRAUBE (com. pes.) registraram 313 espécies também ao longo de um ano de trabalho de campo na floresta tropical pluvial da Serra do Mar, que compõe o Parque Estadual do Marumbi, um ecossistema considerado tradicionalmente como muito rico em aves. Mas estes autores percorreram uma área muito maior que a fazenda Santa Rita, o que revela a alta riqueza específica deste local.

Por se localizar na borda oriental da região dos Campos Gerais, ainda relativamente próxima da Serra do Mar, na fazenda Santa Rita ocorrem espécies que são mais típicas da floresta tropical pluvial (CRACRAFT, 1985) como: Brotogeris tirica (Vieillot, 1817), Cichlocolaptes leucophrys (Jardine \& Selby, 1830), Scytalopus speluncae (Ménétriés, 1835), Piprites pileatus (Temminck, 1822), Myiobius atricaudus Berlepsch, 1888, Phylloscartes oustaleti (Sclater, 1827), Tangara desmaresti (Vieillot, 1819) e Hemithraupis ruficapilla (Vieillot, 1818). Estas espécies não foram registradas no Parque Estadual de Vila Velha a apenas $5 \mathrm{~km}$ a oeste do local de estudo.

Interessante observar que Hemithraupis ruficapilla registrado na $\mathrm{Fa}$ zenda Santa Rita é substituída por H. guira (Linnaeus, 1766) no Parque Estadual de Vila Velha. Assim a Fazenda Santa Rita constitui-se em um limite de ocorrência para estas espécies mais típicas da floresta tropical pluvial. Aparentemente os campos que começam a ocorrer a oeste a partir da Fazenda Santa Rita, constituem uma barreira na distribuição dessas espécies. 
Tabela II. Registros nos habitats (em porcentagem de ocorrência, ver texto) e nos meses de amostragem das espécies de aves observadas na Fazenda Santa Rita. FLO: floresta; CAM: campo; BRE: brejo; PLA: plantação; JAR: jardim; J: janeiro; F: fevereiro; M: março; A: abril; I: maio; U: junho; L: julho; G: agosto; S: setembro; O: outubro; N: novembro; D: dezembro.

\begin{tabular}{|c|c|c|c|c|c|c|c|}
\hline \multirow{2}{*}{ Espécies } & \multicolumn{5}{|c|}{ Habitats } & \multicolumn{2}{|r|}{ Meses } \\
\hline & FLO & CAM & $\mathrm{BRE}$ & PLA & JAR & J F M A & I ULG S OND \\
\hline $\begin{array}{l}\text { Tinamus solitarius } \\
\text { (Vieillot, 1819) }\end{array}$ & 100 & & & & & J F & So \\
\hline $\begin{array}{l}\text { Crypturellus obsoletus } \\
\text { Temminck, } 1815\end{array}$ & 100 & & & & & $\mathrm{~J}$ F MA & I ULG S OND \\
\hline $\begin{array}{l}\text { Rhynchotus rufescens } \\
\text { (Temminck, 1815) }\end{array}$ & & 100 & & & & J F A & I ULGSOND \\
\hline $\begin{array}{l}\text { Nothura maculosa } \\
\text { (Temminck, 1815) }\end{array}$ & & 100 & & & & J F MA & I UL G S OND \\
\hline $\begin{array}{l}\text { Podiceps dominicus (Linch } \\
\text { Arribálzaga, 1766) }\end{array}$ & & & 100 & & & M & \\
\hline $\begin{array}{l}\text { Phalacrocorax olivaceus } \\
\text { (Gmelin, 1789) }\end{array}$ & & & 100 & & & M & \\
\hline Ardea cocoi Linnaeus, 1766 & & & 100 & & & & $\mathbf{U}$ \\
\hline $\begin{array}{l}\text { Casmerodius albus } \\
\text { (Gmelin, 1758) }\end{array}$ & & & 100 & & & & I \\
\hline Egretta thula (Molina, 1782) & & & 100 & & & & $\mathrm{U}$ \\
\hline $\begin{array}{l}\text { Bulbucus ibis (Linnaeus, } \\
1758 \text { ) }\end{array}$ & & 100 & & & & & L G S \\
\hline $\begin{array}{l}\text { Syrigma sibilatrix } \\
\text { (Temminck, 1824) }\end{array}$ & 50 & 83 & & & & $\mathrm{~J}$ F MA & I ULGSOND \\
\hline $\begin{array}{l}\text { Theristicus caudatus } \\
\text { (Boddart, 1783) }\end{array}$ & 58 & 100 & & & & J F MA & I ULGSOND \\
\hline $\begin{array}{l}\text { Amazonetta brasiliensis } \\
(\text { Gmelin, 1789) }\end{array}$ & & & 100 & & & & ON \\
\hline $\begin{array}{l}\text { Cairina moschata } \\
\text { (Linnaues, 1758) }\end{array}$ & & & 100 & & & & G \\
\hline $\begin{array}{l}\text { Sarcoramphus papa } \\
\text { (Linnaeus, 1758) }\end{array}$ & & 100 & & & & & L S \\
\hline $\begin{array}{l}\text { Coragyps atratus } \\
\text { (Bonaparte, 1793) }\end{array}$ & & 42 & 75 & & & J F MA & I ULGSOND \\
\hline Cathartes aura (Spix, 1824) & & 33 & 100 & & & $\mathrm{~J}$ F MA & I U L G S OND \\
\hline $\begin{array}{l}\text { Elanus leucurus (Vieillot, } \\
1818 \text { ) }\end{array}$ & & 14 & 100 & & & $\mathrm{MA}$ & SOND \\
\hline $\begin{array}{l}\text { Leptodon cayanensis } \\
\text { (Latham, 1790) }\end{array}$ & & 100 & & & & & $\mathrm{U}$ \\
\hline $\begin{array}{l}\text { Harpagus diodon } \\
\text { (Temminck, 1923) }\end{array}$ & & 100 & & & & $\mathrm{~F}$ & $\mathbf{U} \mathbf{L}$ \\
\hline $\begin{array}{l}\text { Ictinia plumbea (Gmelin, } \\
\text { 1788) }\end{array}$ & & 100 & & & & $\mathrm{~F}$ & \\
\hline
\end{tabular}

Cont. 
Tabela II. (Cont.).

Espécies

Geranoaetus melanoleucus

(Vieillot, 1819)

Buteo albicaudatus Vieillot, 1816

100

$33 \quad 83$

Butee magnirostris

(Gmelin, 1788)

$83 \quad 25$

Buteo leucorrhous (Quoy \&

Gaimard, 1824)

Heterospizias meridionalis (Latham, 1790)

$10 \quad 100$

Herpetotheres cachinnans

Linnaeus, 1758

100

Micrastur ruficollis

(Vieillot, 1817)

Milvago chimachima

(Vieillot, 1816)

$92 \quad 58$

Polyborus plancus (Miller, 1777)

100

(Bonaparte, 1838)

Falco femoralis Temminck, 1822

Falco sparverius (Swainson, 1837)

100

Penelope obscura

Temminck, 1815

Odontophorus capueira

(Spix, 1825)

Aramides cajanea (Müller, 1776)

Aramides saracura (Spix,

Cariama cristata (Linnaeus, 1766)

Vanellus chilensis (Gmelin, 1789)

Tringa flavipes (Gmelin, 1789)

Bartramia longicauda

(Bechstein, 1812)

Gallinago gallinago

(Vieillot, 1816)

100

100
Meses

FLO CAM BRE PLA JAR J FMA I U L G S O N D

\section{Habitats}

A I G

FM I G ON

25 J FMA I U L G O N D

M

J FMA I G SOND

J F

S

J A I U L S N D

J FMA I ULG S OND

J FMA I ULG S OND

F

F M

$\mathrm{N}$

$$
\text { F A I } \quad \text { G } \quad \text { O } \quad \text { D }
$$

J FMA I G SOND

J FMA I ULG S OND

$\mathrm{F}$

$\mathrm{O}$

25

SOND

$\mathrm{O}$

J FMA I ULG S OND

$\mathrm{O}$

100

J F

$43 \quad 71$
F U L G S O N

Cont. 
Tabela II. (Cont.).

\begin{tabular}{|c|c|c|c|c|c|c|c|}
\hline \multirow{2}{*}{ Espécies } & \multicolumn{5}{|c|}{ Habitats } & \multicolumn{2}{|r|}{ Meses } \\
\hline & FLO & CAM & BRE & PLA & JAR & J F M A & I ULGSOND \\
\hline $\begin{array}{l}\text { Gallinago undulata } \\
\text { (Boddart, 1783) }\end{array}$ & & & 100 & & & & $\mathrm{O}$ \\
\hline $\begin{array}{l}\text { Columba speciosa Gmelin, } \\
1789\end{array}$ & 100 & & & & & & $\mathrm{U} \quad \mathrm{GSON}$ \\
\hline $\begin{array}{l}\text { Columba picazuro } \\
\text { Temminck, } 1813\end{array}$ & 75 & 75 & & & & $\mathrm{~J}$ F M A & I U L G SOND \\
\hline $\begin{array}{l}\text { Columba maculosa } \\
\text { Temminck, } 1813\end{array}$ & 100 & & & & & & SON \\
\hline $\begin{array}{l}\text { Columba cayennensis } \\
\text { Bonnaterre, } 1792\end{array}$ & 100 & 22 & & & & F A & I L G SOND \\
\hline $\begin{array}{l}\text { Columba plumbea Vieillot, } \\
1818\end{array}$ & 100 & & & & & J F A & I $\quad$ SOND \\
\hline $\begin{array}{l}\text { Zenaida auriculata Chubb, } \\
1819\end{array}$ & 27 & 100 & & 18 & 9 & J F M A & U L G S OND \\
\hline $\begin{array}{l}\text { Columbina talpacoti } \\
\text { Temminck, } 1811\end{array}$ & 25 & 42 & & 8 & 67 & J F M A & I ULGSOND \\
\hline $\begin{array}{l}\text { Leptotila verreauxi } \\
\text { Bonaparte, } 1855\end{array}$ & 100 & & & & & J F M A & I U L G SOND \\
\hline $\begin{array}{l}\text { Leptotila rufaxilla Richard } \\
\& \text { Bernard, } 1712\end{array}$ & 100 & & & & & J F M A & I U L G S OND \\
\hline $\begin{array}{l}\text { Geotrygon montana } \\
\text { (Linnaeus, 1758) }\end{array}$ & 100 & & & & & J F M A & SOND \\
\hline $\begin{array}{l}\text { Pyrrhura frontalis (Vieillot, } \\
1817 \text { ) }\end{array}$ & 100 & & & & & J F M A & I U L G SOND \\
\hline $\begin{array}{l}\text { Forpus xanthopterygius } \\
\text { (Ridgway, 1888) }\end{array}$ & 100 & & & & & F A & \\
\hline $\begin{array}{l}\text { Brotogeris tirica (Kuhl, } \\
1820 \text { ) }\end{array}$ & 100 & & & & & J F M A & I U L G S OND \\
\hline $\begin{array}{l}\text { Pionopsitta pileata } \\
\text { (Scopoli, 1769) }\end{array}$ & 100 & & & & & J F M A & I ULG SOND \\
\hline $\begin{array}{l}\text { Pionus maximiliani (Kuhl, } \\
1820 \text { ) }\end{array}$ & 100 & & & & & J F M A & I ULGSOND \\
\hline $\begin{array}{l}\text { Amazona aestiva (Linnaues, } \\
\text { 1758) }\end{array}$ & 100 & 10 & & & 10 & F M & I ULGSOND \\
\hline $\begin{array}{l}\text { Amazona vinacea (Kuhl, } \\
1820 \text { ) }\end{array}$ & 100 & & & & & & I U L G \\
\hline $\begin{array}{l}\text { Coccyzus americanus } \\
\text { (Linnaues, 1758) }\end{array}$ & 100 & & & & & J F M & I U L $\quad S \quad N D$ \\
\hline $\begin{array}{l}\text { Piaya cayana (Linnaues, } \\
\text { 1766) }\end{array}$ & 100 & & & & & J F M A & I ULGSOND \\
\hline $\begin{array}{l}\text { Crotophaga ani (Linnaeus, } \\
1758 \text { ) }\end{array}$ & & & & & 100 & & I \\
\hline
\end{tabular}

Cont. 
Tabela II. (Cont.).

\begin{tabular}{|c|c|c|c|c|c|c|c|c|c|}
\hline \multirow{2}{*}{ Espécies } & \multicolumn{5}{|c|}{ Habitats } & \multicolumn{4}{|c|}{ Meses } \\
\hline & FLO & CAM & BRE & PLA & JAR & J F M A & I U & L G & SOND \\
\hline Guira guira Gmelin, 1831 & & 75 & & & 25 & J F & & & ND \\
\hline $\begin{array}{l}\text { Tapera naevia (Linnaeus, } \\
\text { 1766) }\end{array}$ & 67 & & & 33 & & FM & & & $\mathrm{O}$ \\
\hline Tyto alba (Gray, 1829) & & & & & 100 & & I & & \\
\hline Otus choliba (Vieillot, 1817) & 100 & & & & & A & & & \\
\hline $\begin{array}{l}\text { Pulsatrix koeniswaldiana } \\
\text { (Bertoni \& Bertoni, 1901) }\end{array}$ & 100 & & & & & & & & $\mathrm{~S}$ \\
\hline $\begin{array}{l}\text { Glaucidium brasilianum } \\
\text { (Gmelin, 1788) }\end{array}$ & 100 & & & & & & & & $\mathrm{~N}$ \\
\hline $\begin{array}{l}\text { Speotyto cunicularia } \\
\text { (Temminck, 1822) }\end{array}$ & & 100 & & & & J F M A & I $\mathrm{U}$ & L G & SOND \\
\hline $\begin{array}{l}\text { Ciccaba virgata (Pelzeln, } \\
1863 \text { ) }\end{array}$ & 100 & & & & & $\mathbf{J}$ & & & $\mathrm{S}$ \\
\hline $\begin{array}{l}\text { Strix hylophila Temminck, } \\
1825\end{array}$ & 100 & & & & & J $\quad$ M A & $\mathrm{U}$ & & SOND \\
\hline $\begin{array}{l}\text { Rhinoptynx clamator } \\
\text { (Vieillot, 1807) }\end{array}$ & 100 & & & & & M & & & \\
\hline $\begin{array}{l}\text { Asio flammeus (Vieillot, } \\
\text { 1817) }\end{array}$ & 50 & 50 & & & & $\mathbf{J}$ & & & $\mathrm{O}$ \\
\hline $\begin{array}{l}\text { Nyctibius griseus (Gmelin, } \\
1789 \text { ) }\end{array}$ & 100 & & & & & J F M & & & OND \\
\hline $\begin{array}{l}\text { Lurocalis semitorquatus } \\
\text { (Gmelin, 1788) }\end{array}$ & 40 & 20 & & & 20 & J F & & & OND \\
\hline $\begin{array}{l}\text { Podager nacunda (Vieillot, } \\
\text { 1817) }\end{array}$ & 50 & 50 & & & & $\mathrm{~F}$ & & & $\mathrm{O}$ \\
\hline $\begin{array}{l}\text { Nyctidromus albicollis } \\
\text { (Gmelin, 1789) }\end{array}$ & 100 & & & & & J F & $\mathrm{U}$ & G & \\
\hline $\begin{array}{l}\text { Caprimulgus parvulus } \\
\text { (Gould, 1837) }\end{array}$ & & 100 & & & & J F M A & & L G & SOND \\
\hline $\begin{array}{l}\text { Macropsalis creagra } \\
\text { (Bonaparte, 1850) }\end{array}$ & 100 & & & & & & & & SO \\
\hline $\begin{array}{l}\text { Streptoprocne zonaris } \\
\text { (Shaw, 1796) }\end{array}$ & & 100 & & & & J F M A & I U & L G & SOND \\
\hline $\begin{array}{l}\text { Cypseloides fumigatus } \\
\text { (Streubel, 1848) }\end{array}$ & & 100 & & & & & & & $\mathrm{O}$ \\
\hline $\begin{array}{l}\text { Chaetura cinereiventris } \\
\text { Sclater, } 1862\end{array}$ & & 100 & & & & $\mathrm{~F}$ & & & $\mathrm{O}$ \\
\hline $\begin{array}{l}\text { Chaetura andrei Hellmayr, } \\
1907\end{array}$ & & 100 & & & & & I & L & $\mathrm{ON}$ \\
\hline $\begin{array}{l}\text { Phaetornis squalidus } \\
\text { (Temminck, 1822) }\end{array}$ & 100 & & & & & & & & $\mathrm{O}$ \\
\hline $\begin{array}{l}\text { Phaetornis pretrei (Lesson } \\
\text { \& Delattre, 1839) }\end{array}$ & 100 & & & & & F A & I U & & D \\
\hline
\end{tabular}

Cont. 
Tabela II. (Cont.).

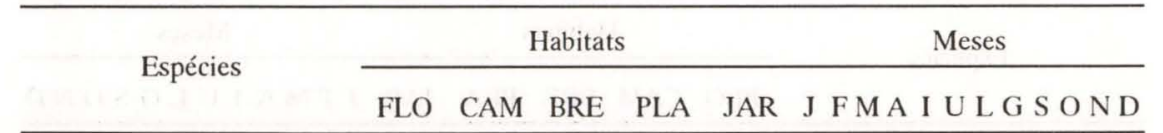

Colibri serrirostris

(Vieillot, 1816)

Stephanoxis lalandi

(Vieillot, 1818)

33

100

Chlorostilbon aureoventris (d'Orb. \& Lafresnaye, 1838)

Thalurania glaucopis

(Gmelin, 1788)

100

Leucochloris albicollis

(Vieillot, 1818)

100

Amazilia versicolor

(Vieillot, 1818)

100

Calliphlox amethystina

(Boddart, 1783)

Trogon rufus Gmelin, 1788

Trogon surrucura Vieillot, 1817

Chloroceryle americana

(Gmelin, 1788)

Baillonius bailloni (Vieillot, 1819)

Ramphastos dicolorus

Linnaeus, 1766

Sundeval, 1866

Temminck, 1825

Colaptes campestris

(Vieillot, 1818)

42

Chrysoptilus melanochloros

(Gmelin, 1788)

100

Piculus aurulentus

(Temminck, 1823)

100

Dryocopus lineatus

(Linnaeus, 1766)

100

Melanerpes flavifrons

(Vieillot, 1818)

100

Veniliornis spilogaster

(Wagler, 1827)

100

Phloeoceastes robustus

(Lichtenstein, 1819)

Dendrocincla fuliginosa

(Vieillot, 1818)

100
67

O N D

A G OND

J F M A

S OND

FMA ULG S N

JFMA U G SOND

$\mathrm{O}$

D

J F A I G S

J FMA LG SOND

F $\quad S O N$

A $\quad \mathrm{SON}$

J FMA I U L G S OND

J F M A I U L G S O N D

J FMA I U L G S O N D

J FMA I U L G S O N D

J FM SOND

J F MA I U L G S O ND

F $\quad$ L G O N

J FMA I U L G S O ND

J F M A I U L G S O N D $\begin{array}{lll} & \text { U G } & \text { G } \\ \text { J } & \text { I SOND }\end{array}$

Cont. 
Tabela II. (Cont.).

Espécies Habitats Meses

FLO CAM BRE PLA JAR J FMA I ULG S OND

Sittasomus griseicapillus

(Vieillot, 1818)

100

Xiphocolaptes albicollis

(Vieillot, 1818)

100

Dendrocolaptes platyrostris

Spix, 1825

Lepidocolaptes angustirostris

(Vieillot, 1918)

100

100

Lepidocolaptes squamatus

(Lichtenstein, 1822)

Lepidocolaptes fuscus

(Vieillot, 1818)

Campylorhamphus

falcularius (Vieillot, 1822)

Clibanornis dendrocolaptoides

(Pelzeln, 1859)

Furnarius rufus (Gmelin,

1816)

Leptasthenura striolata

(Pelzeln, 1856)

Leptasthenura setaria

(Temminck, 1824)

Synallaxis ruficapilla Vieillot,

1819

Synallaxis spixi Sclater, 1856

Synallaxis cinerascens

Temminck, 1823

100

Cranioleuca obsoleta

(Reichenbach, 1853)

Cranioleuca pallida (Wied, 1831)

Anumbius annumbi (Vieillot, 1817)

Syndactyla rufosuperciliata

(Lafresnaye, 1832)

Anabacerthia amaurotis

(Temminck, 1823)

Philydor atricapillus (Wied,

Philydor rufus (Vieillot, 1818) 100

Cichlocolaptes leucophrys

(Jardine \& Selby, 1830)

00

67

J FMA I ULG S OND

J FM I U G SOND

J F M A I U L G S OND

J A I U G SOND

J FMA I ULG S OND

J F M A I ULG S O N D

G

J A I U

8

25 J FMA I ULG S OND

J F M A I U L G S O ND

J FMA I ULG S OND

J FMA I U L G S O ND

J FMA I U L G S OND

J FMA I U L G S O ND

J F M A I U L G S O ND

J FMA I U L G S O ND

59 J FMA I ULG S OND

J FMA I U L G S O ND

J FMA I ULG SOND

L

J FMA I ULG S OND

I S

Cont. 
Tabela II. (Cont.).

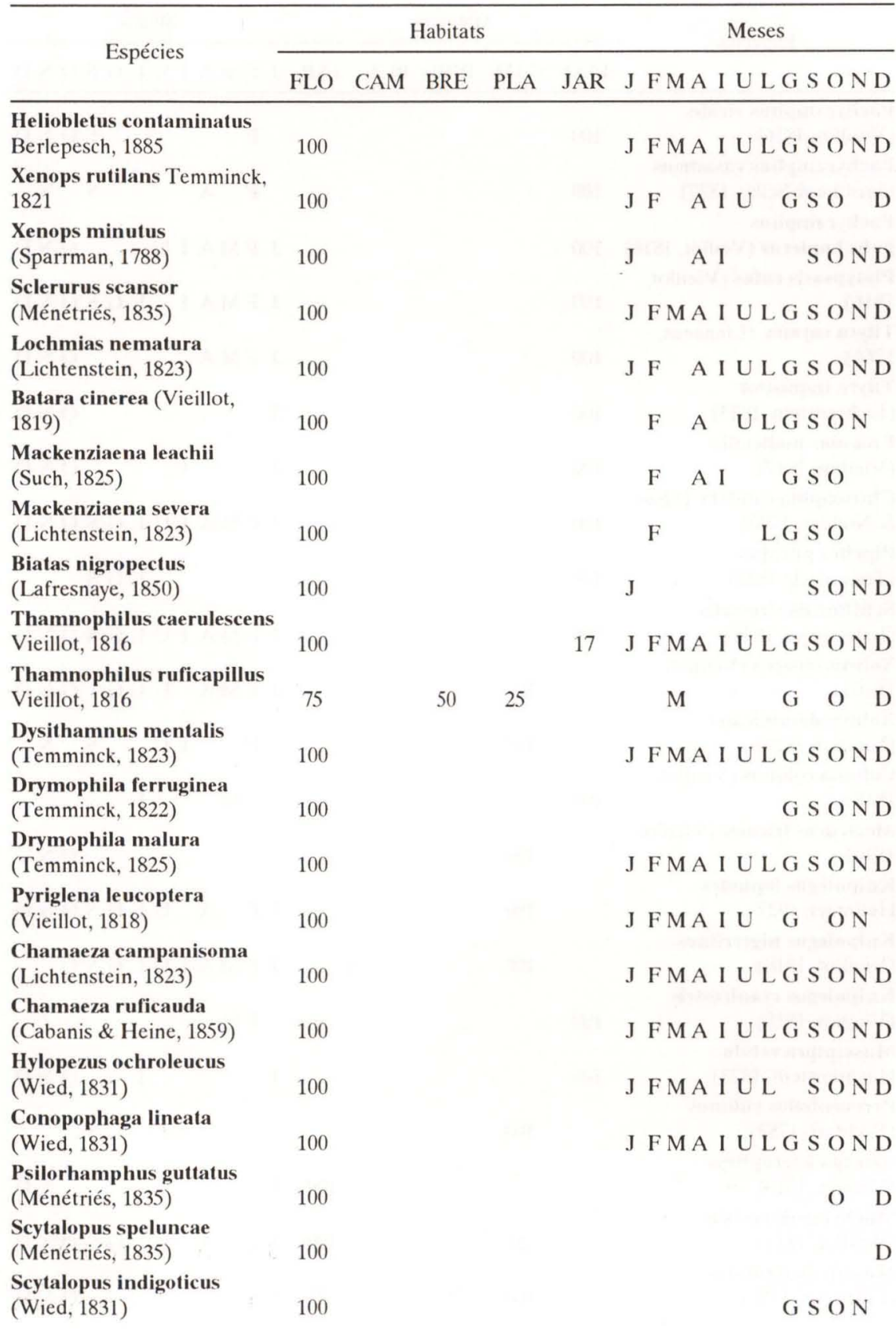

Cont. 
Tabela II. (Cont.).

Espécies

Pachyramphus viridis

(Vieillot, 1816)

100

Pachyramphus castaneus

(Jardine \& Selby, 1827) $\quad 100$

Pachyramphus

polychopterus (Veillot, 1818) 100

Platypsaris rufus (Vieillot, 1816)

100

Tityra cayana (Linnaeus, 1766)

Tityra inquisitor

(Lichtenstein, 1823)

Procnias nudicollis

(Vieillot, 1817)

Chiroxiphia caudata (Shaw

\& Nodder, 1793)

Piprites pileatus

(Temminck, 1822)

Schiffornis virescens

(Lafresnaye, 1838)

Xolmis cinerea (Vieillot,

1816)

Xolmis dominicana

(Vieillot, 1823)

Colonia colonus (Vieillot,

Alectrurus tricolor (Vieillot, 1816)

Knipolegus lophotes

Hellmayr, 1927

Knipolegus nigerrimus

(Vieillot, 1818)

100

100

100

100

100

100

100

100

100

Knipolegus cyanirostris

(Vieillot, 1818)

Muscipipra vetula

(Lichtenstein, 1823)

Pyrocephalus rubinus

(Boddart, 1783)

Satrapa icterophrys

(Vieillot, 1818)

Machetornis rixosus

(Vieillot, 1819)

Muscivora tyrannus

(Linnaeus, 1758)

100

100

100

100

100

100

20

100
Meses

FLO CAM BRE PLA JAR J FMA I ULG S OND

\section{Habitats}

F

S OND

F A

S N

J F M A I U

OND

J F M A I

L G S O ND

J F M A

OND

J

OND

$\mathbf{J}$

I

OND

J F M A I ULG S O ND

L G S

J FMA I ULG S OND

J FMA ULG OND

F I U S N

M L
JF A ULGSOND

J F M A I U L G S O

F M

$\mathbf{J}$

L OND

$\mathrm{S}$

$100 \mathrm{~J}$ N D

100 J F A ULG S O D

20

20 J F

OND

Cont. 
Tabela II. (Cont.).

\begin{tabular}{|c|c|c|c|c|c|c|c|c|}
\hline \multirow{2}{*}{ Espécies } & \multicolumn{5}{|c|}{ Habitats } & \multicolumn{3}{|c|}{ Meses } \\
\hline & FLO & CAM & BRE & PLA & JAR & $\mathrm{J}$ F M A & I U L G & SOND \\
\hline $\begin{array}{l}\text { Tyrannus melancholicus } \\
\text { Vieillot, } 1819\end{array}$ & 83 & 83 & & 17 & 83 & J F M & & OND \\
\hline $\begin{array}{l}\text { Empidonomus varius } \\
\text { Vieillot, } 1818\end{array}$ & 100 & & & & & $\mathrm{~F}$ & & OND \\
\hline $\begin{array}{l}\text { Legatus leucophaius } \\
\text { (Vieillot, 1818) }\end{array}$ & 100 & & & & & & & OND \\
\hline $\begin{array}{l}\text { Megarhynchus pitangua } \\
\text { (Linnaeus, 1766) }\end{array}$ & 100 & & & & & $\mathrm{~J}$ F M A & & OND \\
\hline $\begin{array}{l}\text { Myiodynastes maculatus } \\
\text { (Müller, 1776) }\end{array}$ & 100 & & & & & J F M & & OND \\
\hline $\begin{array}{l}\text { Pitangus sulphuratus } \\
\text { (Linnaeus, 1766) }\end{array}$ & 83 & 58 & 8 & & 58 & $\mathrm{~J} F \mathrm{MA}$ & $\mathrm{I} \cup \mathrm{LG}$ & S OND \\
\hline $\begin{array}{l}\text { Myiarchus ferox Cabanis \& } \\
\text { Gmelin, } 1789\end{array}$ & & 100 & & & & & & $\mathrm{ON}$ \\
\hline $\begin{array}{l}\text { Myiarchus swainsoni } \\
\text { Heine, } 1859\end{array}$ & 100 & & & & & J F M & & OND \\
\hline $\begin{array}{l}\text { Pseudattila phoenicurus } \\
\text { (Pelzeln, 1868) }\end{array}$ & 100 & & & & & $\mathrm{~F}$ & & \\
\hline $\begin{array}{l}\text { Contopus cinereus (Spix, } \\
1825 \text { ) }\end{array}$ & 100 & & & & & & $\mathrm{ULG}$ & SO \\
\hline $\begin{array}{l}\text { Empidonax euleri } \\
\text { (Cabanis, 1868) }\end{array}$ & 100 & & & & & $\mathrm{~J}$ F M A & $\mathrm{L} \mathrm{G}$ & $S O N D$ \\
\hline $\begin{array}{l}\text { Cnemotriccus fuscatus } \\
\text { (Berlepsch, 1908) }\end{array}$ & 100 & & & & & F M A & & $\mathrm{S}$ \\
\hline $\begin{array}{l}\text { Myiobius atricaudus } \\
\text { (Berlepsch, 1888) }\end{array}$ & 100 & & & & & $\mathrm{~F}$ & I & \\
\hline $\begin{array}{l}\text { Myiophobus fasciatus } \\
\text { (Temminck, 1822) }\end{array}$ & 100 & 20 & & & & $\mathrm{~J} F \mathrm{M}$ & & SO \\
\hline $\begin{array}{l}\text { Hirundinea ferruginea } \\
\text { (Gmelin, 1788) }\end{array}$ & & 100 & & & & $\mathrm{~J} F \mathrm{M}$ & & SOND \\
\hline $\begin{array}{l}\text { Platyrinchus mystaceus } \\
\text { (Vieillot, 1818) }\end{array}$ & 100 & & & & & $\mathrm{~J} F \mathrm{FA}$ & I U L G & SOND \\
\hline $\begin{array}{l}\text { Tolmomyias sulphurescens } \\
\text { (Spix, 1825) }\end{array}$ & 100 & & & & & $\mathrm{~J} F \mathrm{MA}$ & I $\cup \mathrm{LG}$ & SOND \\
\hline $\begin{array}{l}\text { Todirostrum plumbeiceps } \\
\text { Lafresnaye, } 1846\end{array}$ & 100 & & & & & $\mathrm{FMA}$ & I $\mathrm{ULG}$ & SOND \\
\hline $\begin{array}{l}\text { Idioptilon nidipendulum } \\
\text { (Wied, 1831) }\end{array}$ & 100 & & & & & $\mathbf{J}$ & G & OND \\
\hline $\begin{array}{l}\text { Myiornis auricularis } \\
\text { (Vieillot, 1818) }\end{array}$ & 100 & & & & & FM A & $\mathrm{ULG}$ & $\mathrm{SON}$ \\
\hline $\begin{array}{l}\text { Hemitriccus obsoletus } \\
\text { (Ribeiro, 1906) }\end{array}$ & 100 & & & & & $\mathrm{~J} F \mathrm{FA}$ & I U L G & $\mathrm{SON}$ \\
\hline $\begin{array}{l}\text { Pogonotriccus eximius } \\
\text { (Temminck, 1822) }\end{array}$ & 100 & & & & & $\mathrm{~J} F \mathrm{MA}$ & I U L G & SOND \\
\hline
\end{tabular}


Tabela II. (Cont.).

Espécies

Phylloscartes ventralis

(Temminck, 1824)

100

Phylloscartes oustaleti

(Sclater, 1827)

Serpophaga subcristata

(Vieillot, 1817)

100

100

9

Serpophaga nigricans

(Vieillot, 1817)

Elaenia flavogaster

(Thunberg, 1821)

100

Elaenia parvirostris Pelzeln, 1868

Elaenia mesoleuca Cabanis \& Heine, 1859

Elaenia chiriquensis

(Pelzeln, 1868)

$50 \quad 50$

Elaenia obscura Zimmer,

1941

100

Myiopagis caniceps

(Swainson, 1837)

100

Camptostoma obsoletum

(Temminck, 1824)

100

Phyllomyias fasciatus

(Thunberg, 1822)

100

Acrochordopus burmeisteri

(Cabanis \& Heine, 1859)

Leptopogon amaurocephalus

(Tschudi, 1846)

Pipromorpha rufiventris

(Cabanis, 1846)

Tachycineta leucorrhoa

(Vieillot, 1817)

Phaeprogne tapera

(Linnaeus, 1766)

Progne chalybea (Gmelin, 1789)

Notiochelidon cyanoleuca

(Vieillot, 1817)

Alopochelidon fucata

(Temminck, 1822)

Stelgidopteryx ruficollis

(Vieillot, 1817)
Meses

FLO CAM BRE PLA JAR J F M A I U L G S O N D

\section{Habitats}

J F MA I ULG S O N D

25

I U L

$\mathrm{O}$

9 J F M A I U L G S O D

100

$\mathrm{O}$

$$
\text { F }
$$

J F M

O N D

J

O N D

J M

$\mathrm{O} \mathrm{N}$

J F

O N D

$$
\text { M A U G S O }
$$

J F M

L G S O N D

J F M

O N D

F M A I U L G

F M A I U L G S O

J F M A I U L G

O N D

100

$100 \mathrm{~J} \mathrm{~F}$

G S O N D

100

F

100

$100 \mathrm{~J} \mathrm{~F}$

G S O N D

100

100

75 J F M A I U L G S O N D

F M

100
O N D

Cont. 
Tabela II. (Cont.).

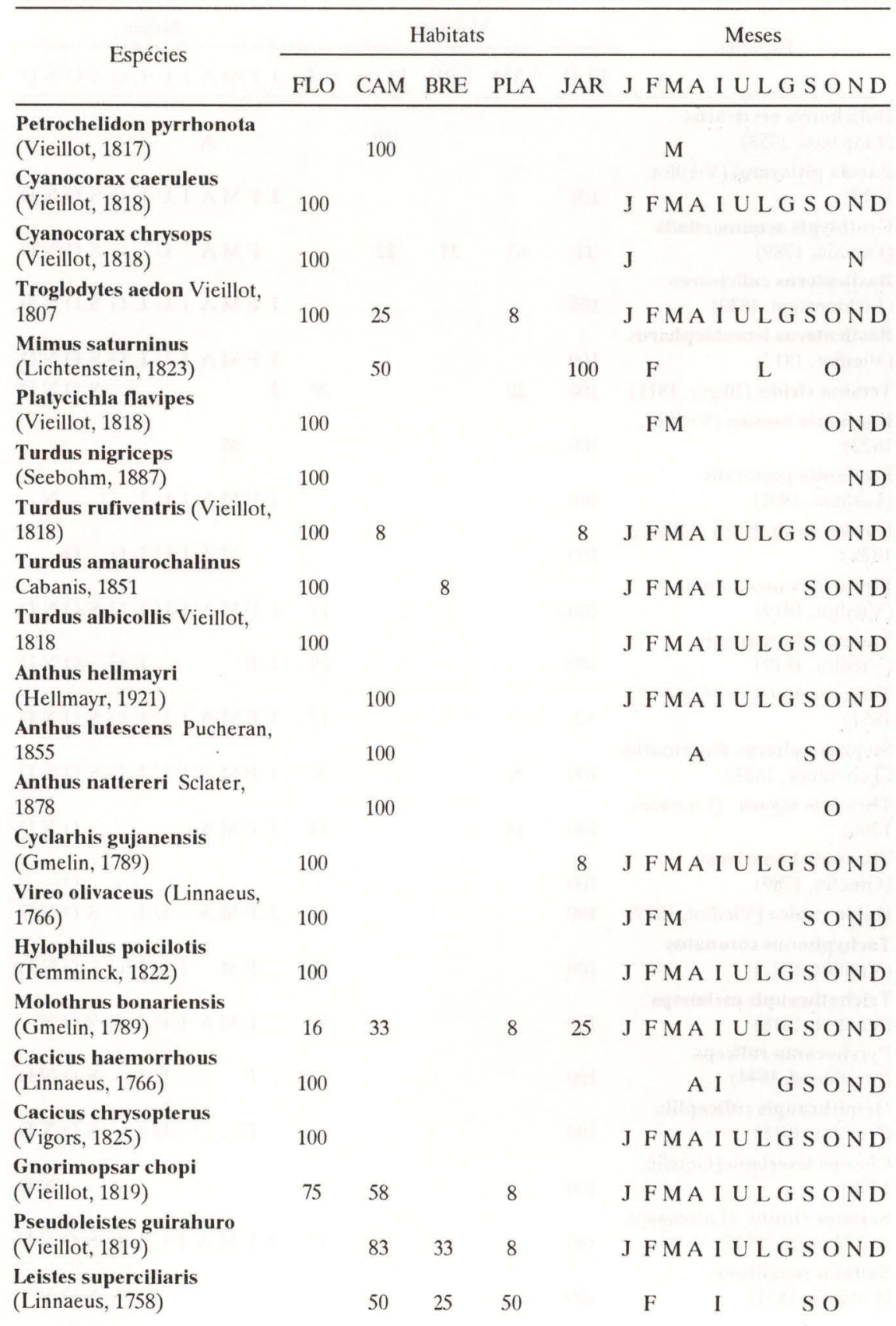

Cont. 
Tabela II. (Cont.).

Espécies

Meses

FLO CAM BRE PLA JAR J FMA I U L G S O N

Dolichonyx oryzivorus

(Linnaeus, 1758)

100

A

Parula pitiayumi (Vieillot, 1817)

100

J F M A I U L S OND

Geothlypis aequinoctialis

(Gmelin, 1789)

$\begin{array}{llll}11 & 67 & 33 & 22\end{array}$

FMA U G SOND

Basileuterus culicivorus

(Lichtenstein, 1830)

100

J FMA I ULG S OND

Basileuterus leucoblepharus

(Vieillot, 1817)

Tersina viridis (Illiger, 1811) 100

20

Euphonia musica (Vieillot,

1822)

100

Euphonia pectoralis

(Latham, 1801)

Euphonia chalybea (Mikan, 1825)

Pipraeidea melanonota

(Vieillot, 1819)

100

Tangara desmaresti

(Vieillot, 1819)

Tangara preciosa (Cabanis, 1851)

Stephanophorus diadematus

(Temminck, 1823)

$100 \quad 8$

Thraupis sayaca (Linnaeus, 1766)

$100 \quad 14$

Thraupis bonariensis

(Gmelin, 1789)

Habia rubica (Vieillot, 1817) 100

Tachyphonus coronatus

(Vieillot, 1822)

Trichothraupis melanops

(Vieillot, 1818)

Pyrrhocoma ruficeps

(Strickland, 1844)

Hemithraupis ruficapilla

(Vieillot, 1818)

Cissops leveriana (Gmelin,

M

J FMA I U L G S OND $20 \mathrm{~J}$

$S O N D$

Saltator similis (Lafresnaye \& d'Orbigny, 1837)

J F M A I U L

N

MA I U L O

17 J FMA I ULG S OND

29 J F

L G OND

17 J FMA I U L G S O ND

8 J FMA I ULG S O N

14 J F M A

O N D

Saltator maxillosus

(Cabanis, 1851)

J FMA UL SOND

10 FM I U L G S OND

10 FMA I U L G S N

F UL SOND

F UL SOND

N D

17 J FMA I ULG S OND

SON

Cont. 
Tabela II. (Cont.),

\section{Espécies}

Habitats

Meses

FLO CAM BRE PLA JAR J FMA I U L G S O ND

Pitylus fuliginosus

(Daudin, 1800)

Cyanocompsa cyanea

(Linnaeus, 1758)

Cyanoloxia glaucocaerulea

(Lafresnaye \& d'Orbigny, 1837)

Volatinia jacarina Linnaeus,

1766

Tiaris fuliginosa (Wied, 1831)

Sporophila caerulescens

(Vieillot, 1817)

Sporophila bouvreuil (Müller,

1776)

Sporophila hypoxantha

(Cabanis, 1851)

Sporophila melanogaster

(Pelzeln, 1870)

Oryzoborus angolensis

(Linnaeus, 1766)

Amaurospiza moesta

(Hartlaub, 1833)

Sicalis citrina (Pelzeln, 1870)

Sicalis flaveola (Linnaeus,

1766)

Sicalis Iuteola (Sparrman,

1789)

Haplospiza unicolor Cabanis,

1851

100

100

100

88

100

33

67

100

60

100

100

50

$\begin{array}{lll}100 \quad 22 & 50 \\ 22\end{array}$

18

18

78

100

100

(Swainson, 1837)

Myospiza humeralis (Bosa,

1792)

Zonotrichia capensis (Müller,

1776)

Emberizoides herbicola

(Vieillot, 1817)

Emberizoides ypiranganus

Ihering \& Ihering, 1907

Donacospiza albifrons

(Vieillot, 1817)

Poospiza nigrorufa

(Lafresnaye \& d'Orbigny, 1837) 100

Poospiza lateralis (Nordmann,

1835)
J

J

G
40

$00 \quad$ M

$\begin{array}{rrr}1350 & \text { J FMA U } \\ \text { FMA } & \text { L }\end{array}$

O N D

O N D

M

$\mathrm{O}$

J F M

N D

I

$\mathrm{ON}$

F M

J FMA G S OND 
Tabela II. (Cont.).

\begin{tabular}{|c|c|c|c|c|c|c|c|}
\hline \multirow{2}{*}{ Espécies } & \multicolumn{5}{|c|}{ Habitats } & \multicolumn{2}{|r|}{ Meses } \\
\hline & FLO & CAM & BRE & PLA & JAR & J FMA I & I U L G S ND \\
\hline $\begin{array}{l}\text { Embernagra platensis } \\
\text { (Gmelin, 1789) }\end{array}$ & & 33 & 83 & & & $\mathrm{~F}$ & $\mathrm{SON}$ \\
\hline $\begin{array}{l}\text { Spinus magellanicus } \\
\text { (Vieillot, 1805) }\end{array}$ & 75 & 75 & & 17 & 58 & J FMA I & I U L G O ND \\
\hline $\begin{array}{l}\text { Passer domesticus } \\
\text { (Linnaeus, 1758) }\end{array}$ & & & & & 100 & & $\mathrm{O}$ \\
\hline
\end{tabular}

Por outro lado Synallaxis frontalis Pelzeln, 1859 registrado no Parque Estadual de Vila Velha não ocorreu na Fazenda Santa Rita. Cyanocorax chrysops (Vieillot, 1818) também muito comum no Parque Estadual de Vila Velha em bandos de 7 a 12 indivíduos só foi registrada uma vez na Fazenda Santa Rita e no reduzido número de dois indivíduos. Portanto o local de estudo também representa um limite de distribuição a leste para estas espécies.

\section{CONCLUSÕES}

A Fazenda Santa Rita apresenta uma rica avifauna, com espécies de campo, de brejo e de floresta (tanto da floresta de araucária como da floresta tropical pluvial), em função da sua localização de transição; a borda oriental dos Campos Gerais. Uma alta porcentagem dessa avifauna é residente. Por outro lado são muitas as espécies migratórias que ocorrem na Fazenda Santa Rita, algumas durante curtos períodos aparentemente utilizando o local como pouso intermediário em seus deslocamentos.

Em função destas características a Fazenda Santa Rita se mostra como local estratégico para conservação da avifauna, ainda mais considerando o estado de degradação ambiental dos Campos Gerais.

AGRADECIMENTOS. Agradecemos aos Profs. Drs. Edwin O’Neill Willis, Wesley Rodrigues Silva, Jacques M. E. Vielliard e Emygdio L. A. Monteiro Filho pelas valiosas críticas e sugestões à tese de doutoramento defendida no Curso de Pós-Graduação em Zoologia da Universidade Federal do Paraná da qual este manuscrito faz parte. Agradecemos também ao Sr. José Carlos Veiga Lopes, proprietário da fazenda Santa Rita, pelo entusiasmo e atendimento durante a realização do trabalho de campo e ao Sr. Gert Hatschbach (Museu Botânico da Prefeitura Municipal de Curitiba) pela identificação das espécies vegetais. O Sr. José Carlos e o zoólogo Pedro Scherer Neto (Museu de História Natural da Prefeitura Municipal de Curitiba) nos forneceram uma lista preliminar de aves da fazenda Santa Rita, não publicada, no que somos siceramente gratos. Em todas as amostragens fomos acompanhados pelo biólogo Roberto Boçon a quem agradecemos sinceramente. 


\section{REFERÊNCIAS BIBLIOGRÁFICAS}

ANJOS, L. DOS 1984. Aspectos etológicos do Myiophobus fasciatus (Aves Tyrannidae) no Estado do Paraná, Brasil. Arq. Biol. Tecnol. 27 (3): 401-405. 1990. Distribuição de aves em uma floresta de araucária da cidade de Curitiba (sul do Brasil). Acta Biol. Par. 19 (1,2,3,4): 51-63.

BELTON, W. 1984. Birds of Rio Grande do Sul, Brazil, Part 1. Bull. Amer. Mus. Nat. Hist. 178: 369-636. 1985. Birds of Rio Grande do Sul, Brazil, Part 2. Bull. Amer. Mus. Nat. Hist. 180: 1-242.

CRACRAFT, J. 1985. Historical biogeography and patterns of diferentiation within the South American avifauna: Areas of endemism. Ornith. Monogr. 36: 49-84.

KLEIN, R.M. 1960. O aspecto dinâmico do pinheiro brasileiro. Sellowia 12: 17-44.

LOPES, J.C. 1982. Curucaca. Inst. Hist. Geog. e Etnog. Par. 14: 215-228.

MEYER DE SCHAUENSEE, R. 1970. A guide to the birds of South America. Wynnewood, Livingston Publ., 498p.

SCHERER-NETO, P. 1982. Aspectos bionômicos e desenvolvimento de Theristicus caudatus (Boddaert, 1783) (Aves, Threskiornithidae). Dusenia 13 (4): 145-149.

SICK, H. 1985. Ornitologia brasileira, uma introdução. Brasília, Editora Universidade de Brasilia, 827p.

VIELLIARD, J.M.E. \& W.R.SILVA. 1990. Nova metodologia de levantamento quantitativo da avifauna e primeiros resultados no interior do Estado de São Paulo, Brasil. Anais do IV Encontro Nacional de Anilhadores de Aves, ed. da Univ. Fed. do Recife.

Recebido em 30.VII.1993; aceito em 25.III.1994. 